\title{
Communicating among robots in the RoboCup Middle-Size League
}

\author{
Frederico Santos ${ }^{1,3}$, Luís Almeida ${ }^{2,3}$, Luís S. Lopes ${ }^{3}$, \\ José L. Azevedo ${ }^{3}$, and M. Bernardo Cunha ${ }^{3}$ \\ 1 DEE - ISEC, Inst. Politécnico de Coimbra, Portugal, fred@isec.pt \\ 2 DEEC - FEUP, Universidade do Porto, Portugal, lda@fe.up.pt \\ 3 IEETA - DETI, Universidade de Aveiro, Portugal, \{lsl,jla\}@ua.pt, \\ mbc@det.ua.pt
}

\begin{abstract}
The RoboCup Middle-Size League robotic soccer competitions pose a real cooperation problem for teams of mobile autonomous robots. In the current state-of-practice cooperation is essential to overcome the opponent team and thus a wireless communication protocol and associated middleware are now fundamental components in the multirobots system architecture. Nevertheless, the wireless communication has relatively low reliability and limited bandwidth. Since it is shared by both teams, it is a fundamental resource that must be used parsimoniously. Curiously, to the best of our knowledge, no previous study on the effective use of the wireless medium in actual game situations was done. In this paper we show how current teams use the wireless medium and we propose a set of best practices towards a more efficient utilization. Then, we present a communication protocol and middleware that follow such best practices and have been successfully used by one particular MSL team in the past four years.
\end{abstract}

\section{Introduction}

The RoboCup Middle-Size League (MSL) [1] has been an effective testpad for cooperative robotics. In fact, beyond all the issues associated with the construction of actual robots for operation in harsh conditions, each team now needs to develop coordinated behaviors to effectively overcome the opponent team. This cooperation is becoming more sophisticated involving the communication of team mates positions, fusion of the ball position, dynamic role assignment, formations and ball passes, among others. The cooperative behaviors are developed on top of an adequate middleware that allows the team members to exchange information. In turn, such middleware relies on a wireless communication protocol.

Despite its importance, however, the wireless communication is known to be less reliable than its wired counterpart with significantly higher bit-error rates, to have limited and variable bandwidth and to be open to the access by other stations not involved in the team, among other undesired phenomena [2]. Nevertheless, the wireless channel must be shared by both teams involved in a 
game, thus becoming a critical shared resource. Curiously, the MSL rules have had not constrains regarding the use of wireless communication. To the best of our knowledge, despite the recurrent problems with wireless communication, no study was ever done to analyze the actual use of the wireless channel in game situations.

Recently, the concern with the wireless communication has increased and the MSL rules are now already including some restrictions on the use of the wireless channel by the participating teams. As a contribution to such effort, in this paper we present an analysis of the actual use of the wireless channel by several MSL teams during the RoboCup 2008 event. We show that there is substantial difference between teams, with some making a parsimonious use of the channel while others use substantial slices of the available bandwidth, few transmiting in a sparse periodic fashion and others sending bursts of data with very short intervals. The patterns of transmission depend on the middleware layer that manages the exchange of information. From the analysis of the communication, we can also infer the kind of middleware being used.

This paper discusses issues related with the wireless communication in the MSL, shows the trend in the MSL rules with respect to the communications, and proposes a few best practices that can improve the general behavior of the wireless channel. Finally, the paper includes a brief description of a specific middleware and communication protocol that follow such best practices and which have been successfully used in MSL competitions in the last four years.

\section{Wireless communication within the MSL}

For several years that the MSL rules already stipulate that the wireless technology to be used is IEEE802.11a/b. The more popular IEEE802.11g technology is not allowed simply because it uses the same band as IEEE $802.11 \mathrm{~b}$ but with fewer, despite larger, frequency channels, which increases the difficulty in channel planning and assignment per competition area to minimize cross-interference [3]. Generally, one channel is assigned to one competition field and both teams playing therein must share it. An attempt is always made to assign non-interfering channels to neighboring fields. Moreover, the communication must be infrastructured, i.e., using access points. Direct ad-hoc communication is not allowed. Other constraints have been included and this year a limitation on the bandwidth allowed to each team was introduced for the first time. Briefly, the MSL rules, concerning the wireless communication currently stipulate:

- IEEE802.11a/b technology

- Infrastructured mode (through Access Point)

- Single $a+$ single $b$ channels per game (each shared by both teams)

- IPv4 addressing within pre-defined networks

- Only unicasts/multicasts (broacasts are forbidden)

- Up to 2.2Mbps bandwidth utilization per team 
The bandwidth limitation was calculated considering the lower bandwidth technology IEEE $802.11 \mathrm{~b}$ (11Mbit/s), which is still used by some teams due to national regulations. This is applied to both types of network, either 'b' and 'a' for fairness reasons.

\subsection{Logs from the MSL at RoboCup 2008}

In order to gather information on how current teams actually use the wireless channel, we monitored the communications during several games of the last edition of RoboCup, in Suzhou. We used one PC with a wireless adaptor configured in monitor mode, which disables filtering and allows receiving all IEEE802.11 packets that arrive at its antenna. The monitoring software was the Wireshark network protocol analyzer and we monitored 6 teams, during periods of approximately 1 minute, randomly taken during the third round-robin games. In all these games all communications took place in IEEE802.11a but the effective bitrates achieve during the competitions varied widely between $6 \mathrm{Mbit} / \mathrm{s}$ and 54 $\mathrm{Mbit} / \mathrm{s}$ with an approximate average of $36 \mathrm{Mbit} / \mathrm{s}$.

Figure 1 shows a set of histograms concerning the distribution of the inter packet intervals related to each team considering the transmissions of all its members as they are effectively transmitted in the wireless medium. We can clearly identify three classes, one of teams 1 and 2 that do some level of traffic spread in the time domain, exhibiting inter-packet intervals that extend up to approximately $80 \mathrm{~ms}$. In the former case, the team uses multicast packets to share information in a producer-consumer fashion. On the other hand, team 2 uses unicasts, with the robots sending exchanging between them in pairs. Then, teams 3 and 4 show a clear dual mode operation with many packets sent in sequence but others sent with longer well defined intervals. Looking in more detail to their logs, we can see that all robots of team 3 transmit periodically and synchronized, with all robots transmiting in sequence and then waiting for a period of approximately $75 \mathrm{~ms}$. Curiosly, this team used IP broadcast frames, which are now banned by the current rules, to exchange information in a producer-consumer fashion. On the other hand, team 4 uses a middleware probably based on a centralized blackboard that resides in one particular station to which all robots send their sensing data periodically, approximate every $150 \mathrm{~ms}$, but often faster. Then, such station carries out some computation, probably sensor fusion, and delivers the result back to the nodes in unicast packets sent in sequence, thus generating a peak of packets sent within a very short interval. Finally, teams 5 and 6 send their traffic in an almost continuous fashion, with very short intervals, leading to numbers of packets that are an order of magnitude higher and to much higher bandwidth utilization levels.

Figure 2 shows the histograms of the packet sizes used by each team in Bytes. Clearly two situations arise, one of teams 1 through 4 that use mainly fixed size packets, in some cases with 2 different sizes, team 1 with average size packets and teams 2, 3 and 4 with relatively small packets, only, and then teams 5 and 6 that use a wide variability of packet lengths with significant use of large $(1.5 \mathrm{kB})$ packets. These teams were the only ones sending bursts of information, 


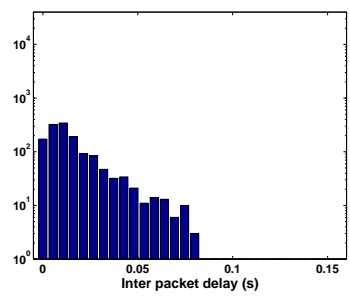

a) Team 1

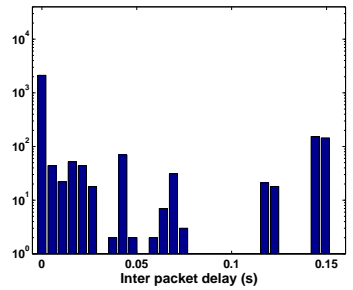

d) Team 4

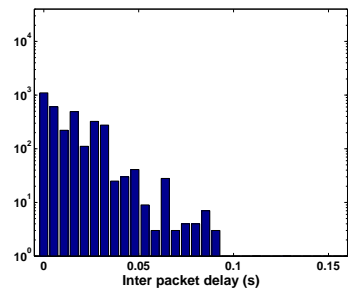

b) Team 2

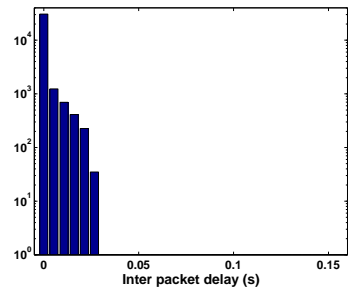

e) Team 5

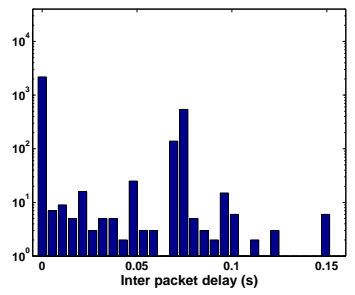

c) Team 3

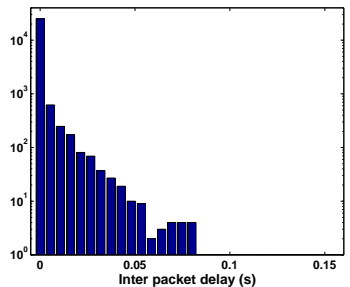

f) Team 6

Fig. 1. Histograms of inter-packet intervals for each team (s)

too. We detected bursts of up to six $1.5 \mathrm{kB}$ packets in the case of team 5 and up to twelve $1.5 \mathrm{kB}$ packets in the case of team 6 . In the IEEE $802.11 \mathrm{a}$ channel used, these bursts, could cause interference of up to $10 \mathrm{~ms}$, approximately, but if an IEEE $802.11 \mathrm{~b}$ channel was used instead, these bursts could imply near $50 \mathrm{~ms}$ delays.

Table 1 shows a summary of the main traffic statistics of the monitored teams, covering inter-packet interval in miliseconds, packet size in bytes, burst size in number of consecutive $1.5 \mathrm{kB}$ packets, total number of bytes transmitted in the monitoring interval and respective approximate utilization in IEEE802.11a/b channels. The traffic classes that were identified in the analysis of the histograms are naturally reflected in this table but the information on the approximate bandwidth utilization of the IEEE802.11a/b channels reveals the huge variations in channel utilization. It is curious to see that team 5 was already using approximately $25 \%$ of the IEEE 802.11 a channel, which corresponds to about $125 \%$ the width of an IEEE $802.11 \mathrm{~b}$ channel. The figures for team 6 are slightly better but still revealling a substantial channel overuse. The other teams use singnificantly lower bandwidths, near 2 orders of magnitude less, which allows them to play without problems among each other using any of the two kinds of channels. According to the current rules, teams 5 and 6 will have to readjust their use of the channel to meet the new $2.2 \mathrm{Mbit} / \mathrm{s}$ limit. One curious detail is the fact that team 6 was using 11 different computers, substantially more that the maximum of 6 robots plus one remote station.

Figure 3 shows the impact that different opponets can have on the timeliness of the transmissions of a robot. In this particular case we used robot1 of team 2 (any other robots yielded similar results) in two games, one against team 1 


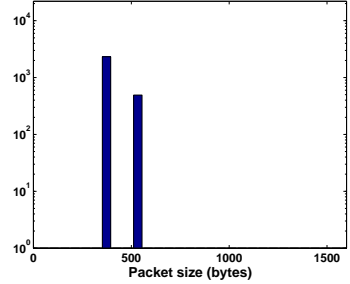

a) Team 1

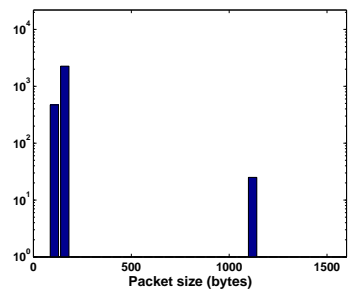

d) Team 4

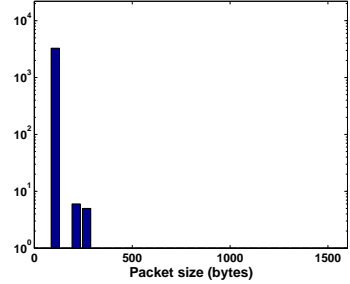

b) Team 2

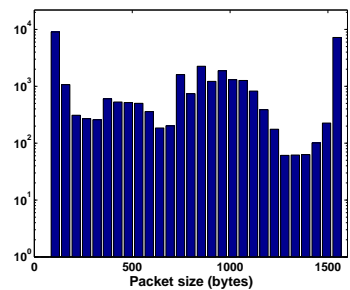

e) Team 5

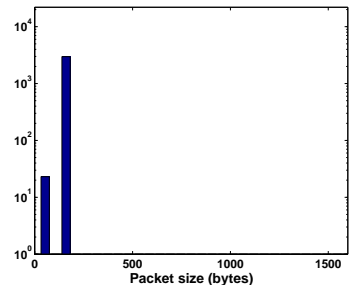

c) Team 3

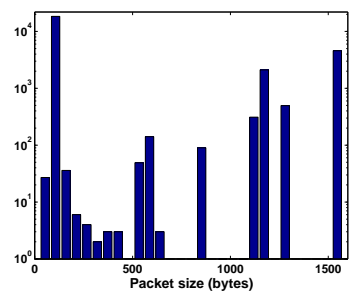

f) Team 6

Fig. 2. Histograms of packet sizes for each team (B)

Table 1. Traffic statistics of 6 MSL teams

\begin{tabular}{|c|c|c|c|c|c|c|c|}
\hline & & Team 1 & Team 2 & Team 3 & Team 4 & Team 5 & Team 6 \\
\hline \multirow{2}{*}{$\begin{array}{c}\text { Inter Packet } \\
(\mathrm{ms})\end{array}$} & avr & 17.74 & 15.20 & 20.03 & 21.72 & 1.74 & 1.90 \\
\hline & std & 17.63 & 14.65 & 33.23 & 48.16 & 3.62 & 4.44 \\
\hline \multirow{2}{*}{$\begin{array}{c}\text { Packet Size } \\
\text { (Bytes) }\end{array}$} & avr & 412.87 & 139.68 & 160.51 & 187.67 & 787.40 & 497.81 \\
\hline & std & 73.66 & 8.03 & 5.59 & 93.77 & 549.09 & 598.36 \\
\hline \multicolumn{2}{|l|}{$\begin{array}{c}\text { Burst Size } \\
(\# 1.5 \mathrm{kB} \text { pk })\end{array}$} & - & - & - & - & 6 & 12 \\
\hline \multirow{2}{*}{\multicolumn{2}{|c|}{$\begin{array}{c}\text { Total kBytes } \\
\% \text { of } \max \end{array}$}} & 1158 & 460 & 480 & 517 & 26154 & 13072 \\
\hline & & 4.43 & 1.75 & 1.84 & 1.98 & 100.00 & 49.98 \\
\hline \multirow{2}{*}{$\begin{array}{l}\text { Bandwidth } \\
\text { utilization }\end{array}$} & $802.11 \mathrm{a}$ & $1.1 \%$ & $0.4 \%$ & $0.5 \%$ & $0.6 \%$ & $25 \%$ & $13 \%$ \\
\hline & $802.11 b$ & $5.5 \%$ & $2.0 \%$ & $2.5 \%$ & $3.0 \%$ & $125 \%$ & $65 \%$ \\
\hline
\end{tabular}




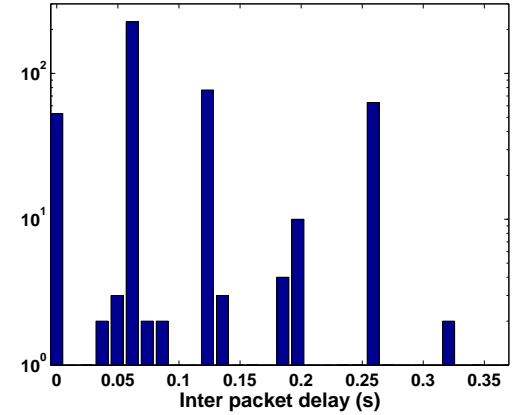

a) Robot 1 of team 2 against team 1

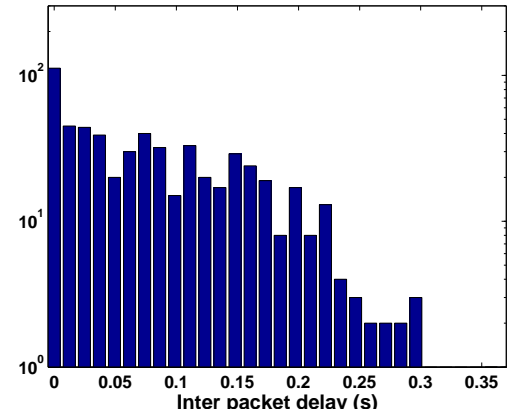

b) Robot 1 of team 2 against team 6

Fig. 3. Inter-packet intervals for one robot of team 2 against teams 1 and 6

that makes a relatively light use of the channel with good separations between packets and, on the other hand, against team 6 that is one of the heavy users of the channel. The figures clearly illustrate the impact of playing against a heavy channel user team. When playing against team 1, the traffic pattern shows a significant regularity, indicating neglectable interference. However, the same robot playing against team 6 shows a significant change in the traffic pattern with a loss of the previous regularity and wide spread (strong jitter) of the inter-packet intervals, with a strong peak close to zero, meaning that many packets are strongly delayed and accumulated at the network interface, being then transmitted in a burst.

Finally, it is important to refer that in these games the traffic external to the competition, including beacon frames from the AP, packets from other teams that were not playing, unknown packets, etc., was always neglectable, representing less than $1 \%$ of the channel bandwidth. Another observation, during a different game than those monitored, was the use of raw (non-IP) packets by another team, which is also in violation with the current rules.

\subsection{Problems and solutions}

In MSL, and probably in other RoboCup competitions as well, the wireless communication has always been a source of concerns, given the frequent occurrence of problems. These were of diverse kinds and we could, in a simplified approach, classify them in four categories: infrastructure configuration, team communications configuration, lack of policing and channel overuse by teams.

- Infrastructure configuration. This category includes the cases in which the planning of the APs placement and channel assignment was non-optimal, frequently caused by constraints of the physical space in which the competitions must be layed out. Since it may be impossible to completely avoid this situaiton, we may have to live with a certain level of background interference, corresponding to an effective lower available channel bandwidth. 
Another problem we experienced, was the interference with pre-installed WLANs for general Internet access, which should have been switched off. This is a relevant issue that local organizers sometimes overlook.

- Team communications configuration. This has been one of the most common sources of problems due to frequent poor knowledge of the wireless communications technology. In fact, it is still common to find teams that bring their own APs and connect them freely in the team work area, often close to competion fields. Other times, the teams use erroneous configurations without being aware (e.g. ad-hoc mode), or send bursts of short packets overloading the network interfaces of the opponent team and causing some device drivers to crash, disabling communications and preventing a team from playing. Without an accurate analysis of the situation, the wrong team can be disqualified due to inability to play.

- Lack of policing. Despite being generally permissive, the MSL rules have dictated certain constraints for some time. Unfortunately, there was always a lack of policing to verify their effective application and enforcing them. In some years, the local organization has hired a specialized company to monitor and control the use of the wireless channels. However, even in such cases it was difficult to mitigate all undesired situations, given their diversity, the number of wireless-enabled computers in the area, and the lack of rules compliance verifications for the teams. We believe that the current rules improved substantially in this aspect by including a network monitor in the games communication architecture and requesting teams to carry out an a priori communications check as a mandatory step for admission to the competitions. Moreover, the network monitor will also allow detecting situations that often occur in which teams in the work area keep their wireless interfaces open and transmitting, causing interference, as well as situations reported in the previous section of teams that use more computers than allowed, that use logical broadcasts and non-IP traffic. Nevertheless, it is still important that the organization is prepared to seek for spurious sources of interference, in case of need. This might require the use of a specific wireless channel monitoring device that provides information on the channel status, not only at the network protocol level (transmitted valid packets) but also at the physical level (bit-error rate, spurious packet fragments, medium spectral analysis, ...).

- Channel overuse by teams. Even without spurious interference, when the channel utilization approaches high values the channel performance deteriorates in terms of packet transmission delays and packet losses due to increased collisions and channel saturation. These delays and losses have a direct negative impact on the quality of the cooperating behaviors given their real-time character, mainly when they involve feedback control over the wireless channel. As it became clear in the previous section, in 2008 some teams were using, alone, more bandwidth than the 'b' technology can provide! This will hopefully be avoided in 2009 given the bandwidth limitation imposed by the new rules. Nevertheless, beyond the channel permanent saturation, transient saturation must also be considered and prevented, such as 
caused by bursts transmitted by the same station, e.g., file transfers. These can also cause a transient increase in packet delays and losses suffered by the opposing team that can harm the performance of its cooperative applications. To prevent these situations the teams must adhere to some kind of control of the consecutive amount of data that each of their robots transmits in an agreed interval of time. On the other hand, detecting such situations requires monitoring the traffic with increased temporal resolution.

As it was clear with the previous discussion, most of the problems that existed in the past can be solved or strongly attenuated with adequate restrictions on the use of the wireless channel and an effective policing of the channel utilization. Nevertheless, it is interesting to quickly analyze certains misconceptions that hindered the deployment of such solutions:

- No need for restricting teams transmissions. Ideally, if the channel bandwidth was infinite and there was no mutual interference between the competiting teams, restricting the teams transmissions would make no sense. However, that is not the case and finite bandwidth and mutual interference are facts that need to be considered. Then, while some teams do a parsimonious use of the channel, others exist that use substantial amount of bandwidth, often in a bursty way, with negative impact on the timeliness of the transmissions of the opposing team, as shown in the previous section, and consequently on the performance of its cooperative behaviors. Thus, some form of restriction that considers both bandwidth and bursts must be enforced.

- Larger bandwidth solves the problem. Unfortunately, just increasing the available bandwidth alone, as when moving from IEEE802.11b (11Mbit/s) to IEEE802.11a $(54 \mathrm{Mbit} / \mathrm{s})$, is not a self-sustained solution and tends to generate wasteful patterns in bandwidth utilization. Such kind of simplistic solutions is always transitory and end up coming back to the same problem but with a larger magnitude. This trend was verified with two of the teams shown in the previous section.

- Use a technology with QoS support. In order to provide better support to time-sensitive traffic with respect to non-time-sensitive one in WLANs, a new standard was recently proposed, namely IEEE802.11e. Similarly to the original protocol, it includes two channel access policies, one that is distributed (EDCA) and another one that is controlled (HCCA). The former is the one that is starting to be accessible commercially while the latter has not received significant adherence by equipment manufacturers so far. Unfortunately, the latter is also the one that could bring more advantages to the RoboCup environment since it allows creating isolated channels with negotiated bandwidth, thus without mutual interference. The former just creates prioritized traffic classes, which does not help since, within a game, one team cannot be prioritized with respect to the other and rules would still be needed to guarantee fairness when sharing the same priority class. Moreover, there would be no guarantee that other external sources of interference would not transmit at the same or higher priority level, thus not 
avoiding the interference problem. Since it is not clear whether equipment supporting HCCA will ever be available due to market reasons, and its expected higher cost, it seems unnecessary to change the current technology and worth working on enforcing appropriate bandwidth sharing policies and mechanisms.

- No need for technical verifications. Ideally, teams should verify and enforce compliance of their equipment with the rules. However, in some cases, particularly with the wireless communication technology due to its idiosyncrasies, the teams often lack the knowledge to adequately enforce the needed configurations. Without technical verifications before the actual competitions, those problems will be discovered in the game, only, and will be hard to diagnose correctly.

\subsection{Further improvements}

We believe that the recent change in the MSL rules, in what concerns communications, positively addressed most of the issues discussed above and constitutes a clear step toward a reliable and efficient use of the wireless medium. Namely, the architecture with predefined IP addresses and with a network monitor, the enforcement of technical verifications specifically concerning the wireless communication as a pre-requisite to admission to the competitions and the bandwidth limitation per team will provide the needed tools to reduce the problems that have been hindering the league. However, we also believe that a further restriction is still needed to definitely increase the robustness of the communications, namely to bound the burstiness of the teams transmissions. This will enforce an adequate permeability of the traffic patterns allowing the adequate interleaving of packets from different sources resulting in lower transmission latencies. This effect is well known in the real-time communications community and can be enforced with techniques that limit the amount of traffic sent within a predefined time interval, such as the leaky bucket [4], or simply using a periodic transmission pattern with relatively small amounts of information [5].

Moreover, the bandwidth limitation of $2.2 \mathrm{Mbit} / \mathrm{s}$ is hard to apply by the teams because of two reasons. On one hand, it is hard to convert actual transmitted bytes to bandwidth due to the idiosyncrasies of the wireless communication (dynamic bit-rate). On the other hand, bandwidth is a compound metric that represents an average amount of information sent per unit of time. If no interval of time is specified, it is still possible for a team to block the channel with a long burst and then compensate with some time of silence and still meet the average bandwidth stipulated in the rules. This has a significantly different impact than using the same bandwidth frequently transmitting short amounts of information. Thus, we believe the rules should not provide a limitation in bandwidth but in number of bytes per given interval of time, which is a metric that teams can easily work with. In particular, we believe that a limitation similar to $1.5 \mathrm{kB}$ per $20 \mathrm{~ms}$ interval are reasonable values to work with for three reasons. Firstly, they correspond approximately to the current target of $20 \%$ channel utilization 
in the MSL rules for normal game situations with an IEEE802.11b channel. Secondly, they are very easy to enforce by any team, since a periodic process of $20 \mathrm{~ms}$ is easily achieved with general purpose operating systems (i.e., no special real-time support is needed) and the $1.5 \mathrm{kB}$ data fits in a single packet, without need for fragmentation. Thirdly, the period of $20 \mathrm{~ms}$ is normally adequate to the dynamics of the cooperative behaviors. Nevertheless, it is still possible to send $750 \mathrm{~B}$ every $10 \mathrm{~ms}$ or even $375 \mathrm{~B}$ every $5 \mathrm{~ms}$ if faster reactivity is needed, without violating the same constraint. Note, equally, that, as demonstrated in this paper with the logs that were carried out, such restriction can be easily policed with a common PC-based/Wireshark network monitor, or the specific monitor that the new MSL rules now refer to.

Finally, the middleware used also has a significant impact. For example, using multicasts in a producer-consumer style allows a faster dissemination of the information, with better synchronization, for four or more stations, on average. A preliminary study of the effect of using multicast/broadcast packets versus unicast ones in a multi-robot scenario is shown in [6]. Direct pair-wise exchange of information, in a peer-to-peer fashion, tends to generate much more traffic for disseminating the same information. Similarly, the use of a central blackboard used in a client-server fashion requires about twice the transmissions than a corresponding producer-consumer model.

As a summary of best practices, we suggest:

- For the teams:

- using a middleware that minimizes transmissions, e.g., with multicasts,

- using a periodic transmission pattern that is permeable to the traffic from the opponent team,

- using low bandwidth cooperation approaches that can work well with the exchange of reasonably small amounts of data,

- verifying the wireless communications compliance with the rules before the actual competitions,

- not connecting APs that are not under the control of the organization,

- not transmitting wireless traffic during competitions while in the neighborhood of the fields.

- For the organization:

- carry out the adequate planning of APs and channels,

- making sure that any pre-installed WLAN for general Internet access in the compound is switched off,

- enforcing technical verifications of the wireless communications,

- deploying the communications architecture in the 2009 MSL rules,

- particularly carrying out the traffic policing using the network monitor,

- having a specific network analyzer at hand, capable of providing information on the status on the physical channel.

\section{RTDB middleware and reconfigurable TDMA protocol}

An example of a pair middleware / communication protocol that we consider that fulfills the best-practices referred above for the teams side, is the Real-Time 


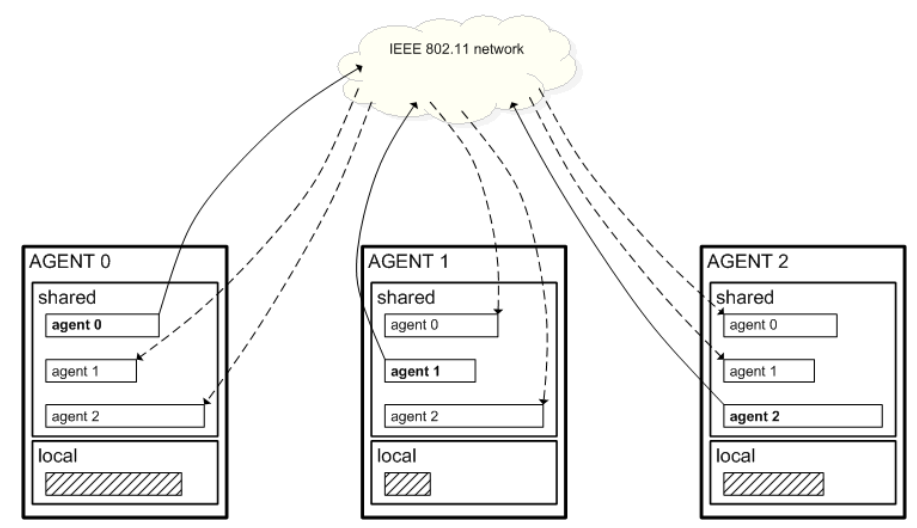

Fig. 4. Each agent transmits periodically its subset of state data that might be required by other agents

DataBase (RTDB) middleware, originally developed in 2004 [7], and the reconfigurable adaptive-TDMA protocol described in [8]. The RTDB middleware targets providing an efficient and timely support for the fusion of the distributed perception and the development of coordinated behaviors by providing a seamless access to the complete team state using a distributed database that is partially replicated in all team members. This database contains images of both local and remote state variables that are accessed locally with fast non-blocking operations. The images of the remote data are updated autonomously in the background by the wireless communication protocol, with each team member disseminating its shared state variables using one multicast packet periodically, at a refresh rate that is adapted to the data dynamics (Fig. 4).

The protocol works on top of IEEE802.11 with an innovative layer that enforces a TDMA-like synchronization among the team members. This synchronization aims at avoiding collisions within the team and it is based on the reception instants of the packets from the other team members, without using clock synchronization. The adaptive nature of the protocol arises from its ability to rotate the phase of the TDMA round to avoid periodic interference patterns. The TDMA round period is the only parameter set off-line and it is called team update period $\left(T_{\text {tup }}\right)$, setting the responsiveness and the temporal resolution of the global communication. It is, thus, an application requirement. $T_{t u p}$ is divided equally by the number of currently active team members generating the TDMA slot structure. This structure is reconfigured dynamically everytime a node leaves (e.g., crashes) or joins the team. The stations transmit at the begining of their slots, thus maximizing the interval between their transmissions and increasing the resilience of the protocol. This middleware plus communication protocol are fully distributed and need minimal configuration facilitating its deployment. 


\section{Conclusions}

Cooperative robotics is an exciting field that has received growing attention in recent years. RoboCup robotic soccer competitions, including the MiddleSize League (MSL), are examples of initiatives that have been fostering the research in that domain. However, the actual cooperation relies on effective wireless communication and middleware layer, but achieving the desired effective use of the wireless channel is not trivial and requires imposing rules to its fair sharing, enforcing them and requesting cooperation from the teams to comply with them. In this paper we showed a first analysis of the wireless channel usage within MSL during actual games. We showed that there is a substantial variability in the way teams communicate and that, in several cases, the use practices are not compliant with the current rules, making it evident the need for traffic policing. We then deduced a set of recommendations that we believe help achieving the desired effective use of the wireless channel. Then the paper briefly presented one specific middleware and communication protocol that comply with the suggested recommendations and have been successfully used in MSL competitions in the last four years.

\section{References}

1. RoboCup: website. http://www.robocup.org

2. Willig, A., Matheus, K., Wolisz, A.: Wireless Technology in Industrial Networks. In: Proceedings of the IEEE. Volume 93. (June 1995) 1130-1151

3. IEEE: IEEE Standard for Information Technology-Telecommunications and Information Exchange Between Systems-Local and Metropolitan Area Networks-Specific Requirements - Part 11: Wireless LAN Medium Access Control (MAC) and Physical Layer (PHY) Specifications. IEEE Std 802.11-2007 (Revision of IEEE Std 802.111999) (December 2007) C1-1184

4. Carpenzano, A., Caponetto, R., Bello, L.L., Mirabella, O.: Fuzzy Traffic Smoothing: An Approach for Real-Time Communication over Ethernet Networks. 4th IEEE International Workshop on Factory Communication Systems, 2002 (2002) 241-248

5. Santos, F., Almeida, L., Pedreiras, P., Lopes, L.S., Facchinetti, T.: An Adaptive TDMA Protocol for Soft Real-Time Wireless Communication Among Mobile Autonomous Agents. In: Proceedings of the WACERTS04 Workshop on Architectures for Cooperative Embedded Real-Time Systems, Lisbon, Portugal (December 2004)

6. Santos, F., Almeida, L.: On the Effectiveness of IEEE802.11 Broadcasts for Soft Real-Time Communication. In: Proceedings of the RTN'05 - 4th International Workshop on Real-Time Networks, Palma de Mallorca, Spain (July 2005)

7. Almeida, L., Santos, F., Facchinetti, T., Pedreiras, P., Silva, V., Lopes, L.S.: Coordinating Distributed Autonomous Agents with a Real-Time Database: The CAMBADA Project. In Aykanat, C., Dayar, T., Korpeoglu, I., eds.: ISCIS. Volume 3280 of Lecture Notes in Computer Science., Springer (2004) 876-886

8. Santos, F., Almeida, L., Lopes, L.S.: Self-Configuration of an Adaptive TDMA Wireless Communication Protocol for Teams of Mobile Robots. In: Proceedings of the 13th IEEE International Conference on Emerging Technologies and Factory Automation, Hamburg, Germany (2008) 